\begin{tabular}{|c|c|}
\hline Title & Comparison of phosphocreatine concentration in the human masseter and medial pterygoid muscles by 31P-CSI \\
\hline Author(s) & Kanay ama, Takeo \\
\hline Citation & 北海道大学. 博士(歯学) 甲第5471号 \\
\hline Issue Date & $2001-03-23$ \\
\hline DOI & $10.11501 / 3182269$ \\
\hline DOC URL & http:/hdl.handle.net/2115/51498 \\
\hline Type & theses (doctoral) \\
\hline Note & $\begin{array}{l}\text { Short title: Metabolism of Masseter and Medial Pterygoid muscles 共著者あり。共著者名:Minowa K., Inoue N., } \\
\text { Y amaguchi T., T amura T., Y oshida S., Kawasaki T. }\end{array}$ \\
\hline File Information & 000000399846.pdf \\
\hline
\end{tabular}

Instructions for use 
Comparison of phosphocreatine concentration in the human masseter and medial pterygoid muscles by 31P-CSI

T. Kanayama, K. Minowa, N. Inore, T. Yamaguchi, T. Tamura,

S. Yoshida and T. Kawasaki 


\section{Comparison of phosphocreatine concentration in the human masseter and medial pterygoid muscles by ${ }^{31} \mathrm{P}$-CSI}

T. Kanayama ${ }^{\prime *}$, K. Minowa ${ }^{2}$, N. Inoue ${ }^{4}$, T. Yamaguchi ${ }^{4}$, T. Tamura, S. Yoshida ${ }^{3}$ and T. Kawasaki' $^{1}$

${ }^{1}$ Department of Prosthetic Dentistry I, ${ }^{2}$ Dental Radiology, and ${ }^{3}$ Oral Anatomy I, School of Dentistry, Hokkaido University, N13 W7, Kita-ku, Sapporo 060-8586, Japan

${ }^{4}$ Special Clinic for Specific Disorders, Dental Hospital, Hokkaido University, N13 W6, Kita-ku, Sapporo 060-8586, Japan; " corresponding author

T. Kanayama: Department of Prosthetic Dentistry I, School of Dentistry, Hokkaido University, N13 W7, Kita-ku, Sapporo 060-8586, Japan

E-mail: kanayama@den.hokudai.ac.jp

Tel. $+81-11-706-4221$

Fax. +81-11-706-4928

Short title: Metabolism of Masseter and Medial Pterygoid muscles

Key words: masseter, medial pterygoid, spectroscopy ( $\left.{ }^{31} \mathrm{P}-\mathrm{MRS}\right)$, chemical shift imaging (CSI), metabolism. 
Summary. The aim of this study is to compare phosphocreatine ( $\mathrm{PCr})$ concentrations of human masseter and medial pterygoid muscles by a recently developed localized MRS method, Chemical Shift Imaging (CSI). The characteristic spectra of phosphorus metabolites including $\mathrm{PCr}$ and $\beta$-ATP from the superficial part of the masseter (SM) and the deep part of the masseter (DM) and the medial pterygoid muscles (MPt) from 11 volunteers, 20-27 years-old were obtained. The study clearly demonstrated higher $\mathrm{PCr} / \beta$-ATP in the SM and MPt than in the DM both in mean values $(\mathrm{p}<0.01)$ and in individual subjects. The results indicate that SM and MPt are power producers. There were no significant differences in the mean values of the PCr/ $\beta$-ATP ratios in SM and MPt, however, the PCr/ $\beta$-ATP ratios varied individually and the subjects could be divided into three distinct groups: values of MPt higher than SM (group A, 4 subjects); values of MPt almost equal to SM (group B, 3 subjects); and values of MPt lower than SM (type C, 4 subjects). There appears to be a close relationship between the $\mathrm{PCr}$ content as determined in the groups here and occlusal guidance. 


\section{Introduction}

Bioptical methods are conventionally used to measure the concentrations of so-called high-energy phosphates in muscles, however, these methods require tissue samples from the subjects. Recently, ${ }^{31} \mathrm{P}-\mathrm{Magnetic}$ Resonance Spectroscopy $\left({ }^{31} \mathrm{P}-\mathrm{MRS}\right)$ has been widely applied to the study of the muscular metabolism (Meyer et al., 1982; Boicelli et al., 1989; Bernus et al., 1993), because it provides noninvasive measurements of phosphocreatinine ( $\mathrm{PCr}$ ) concentrations in muscles. The $\mathrm{PCr}$ is an energy-rich phosphate in muscles, and its concentration in the muscle is considered to relate to the muscle contractile force (Park et al., 1988; Vandenborne et al., 1995).

Formerly, ${ }^{31} \mathrm{P}-\mathrm{MRS}$ studies had been performed with surface-coils and simple pulse-acquire sequences (Taylor et al., 1983; Arnold et al., 1985), however the lack of sensitivity and signal localization limits examination to only the superficial site. To overcome this limitation, a new localized MRS method, Chemical Shift Imaging (CSI), has been developed to examine deeper parts of the muscle. This new method has succeeded to obtain clear signals from human masseter muscle, and has allowed measurements of the different contents of $\mathrm{PCr}$ in the deep (DM) and superficial parts (SM) of the masseter (Kanayama et al., 2000).

The medial pterygoid muscle (MPt) is known as a deep counterpart to masseter, and it acts to elevate the mandible with the masseter. However the MPt has not been studied with MRS because of the inaccessibility of the muscle. The present study examined the $\mathrm{PCr}$ contents of the medial pterygoid muscle, and compared the results with the deep and superficial part of the masseter. 


\section{Materials and Methods}

\section{Subjects}

Eleven male volunteers aged 20-29 years-old participated in the study. Local ethics committee approval and informed consent are obtained for each subject. All subjects had full dentition, and were free from orofacial pain or pathologies of the masticatory system.

\section{Magnetic Resonance examination}

Magnetic Resonance experiments were performed on a Siemens Magnetom Vision whole-body $1.5 \mathrm{~T} \mathrm{MRI/MRS}$ system with a $14 \mathrm{~cm}$ diameter, dual-tuned, $64 / 25 \mathrm{MHz}$ ${ }^{1} \mathrm{H} /{ }^{\beta 1} \mathrm{P}$, radio frequency surface-coil. First, the coil was shimmed using the proton frequency until the water peak dropped down to $0.5 \mathrm{ppm}$ full width half maximum (FWHM), then survey serial Magnetic Resonance images from orbita to mandible were obtained to verify the position of VOI over each part of the Mas and MPt. The DM and SM were distinguished by the existence of aponeurosis between the two (Minowa et al., 1998). The slice selection was made by slice selective gradient in the long axis direction for masseter and in the sagittal axis direction for medial pterygoid, and 2D-CSI was performed with each of 16 phases encoding in two dimensions. Slice thickness was 30 $\mathrm{mm}$ and the focus of view (FOV) was $320 \mathrm{~mm}$. The voxel dimensions after k-space zero filling were $1 \times 2 \times 3 \mathrm{~cm}^{3}$.

Figure 1 shows the cross-sectional MR images of DM, SM, and MPt. All measurements were performed on almost the same portion as Fig. 1 in each subject. Raw data were acquired with a repetition time of $330 \mathrm{~ms}$ by 9 transients, resulting in a measurement duration of $12 \mathrm{~min}$ and $30 \mathrm{sec}$. Before Fourier transformation the sum of the free 
induction decays (FIDs) were multiplied by an exponential with decay chosen to correspond to a $6.4 \mathrm{~Hz}$ line broadening. After the Fourier transformation, all spectra were adjusted with zero- and first-order phase corrections. The integrals of the resonance areas from the $\mathrm{PCr}$ and $\beta$-ATP peaks, which represent the amounts of phosphate in vivo MRS, were measured by a fitting procedure using lorentzian line shapes.

This study evaluated the PCr level in the muscle with the use of PCr/ $\beta$-ATP ratios. Because (1) traditionally, human ${ }^{31} \mathrm{P}$-spectra are quantified in relative terms only, (2) PCr/ATP is considered as an index of the energetic state (Neubauer et al., 1998), and (3) the peak of $\beta$-ATP was higher in all ATP, and most stable in the spectra (Marcel, 1995: Kanayama et al.. 2000). The data were subjected to a Sheffe's F test with the level of significance set at $\mathrm{P}<0.05$.

Prior to the start of the examination, repeated measurements on a single individual were carried out 6 times over 6 weeks to determine the reproducibility of the CSI results on each muscle (Fig. 2), then the CSI examinations were performed with the 11 subjects. The relative SE [(SE/mean)x100] of the PCr/ $\beta$-ATP in the SM, DM, and MPt for the 6 measurements were $0.8,1.3$, and $1.1 \%$ respectively.

\section{Result}

Fig. 3 shows typical phosphorus MR spectra obtained from each muscle at rest. Several resonances reflecting the high-energy phosphate concentrations, such as $\beta-\mathrm{ATP}, \mathrm{PCr}$, and $\mathrm{Pi}$ could be detected. The phosphomonoester (PME) and phosphodiester (PDE) peaks, which reflect sugar-phosphates and membrane phospholipids metabolites, could also be observed. Although it is difficult to establish the absolute phosphate content by 
these data alone, the peak and integral values of $\mathrm{PCr}$ in the DM were lower than in SM and MPt. The values of $\beta$-ATP were very similar in all three.

The mean values of PCr/ $\beta$-ATP were 4.68 in the SM, 3.51 in the DM, and 4.65 in the MPt (Table). The PCr/ $\beta$-ATP ratio was significantly $(\mathrm{p}<0.01)$ higher in the SM and MPt than in the DM, while no significant differences were found between SM and MPt.

Fig. 4 shows the integral values of $\mathrm{PCr} / \beta$-ATP in the three muscles for all the 11 subjects. Similar to the results of the mean values described above, the values of $\mathrm{PCr} / \beta$-ATP ratios in all subjects were higher in the SM and MPt than in the DM except for one subject. However, the values of the PCr/ $\beta$-ATP ratios between the SM and MPt varied individually. The subjects were divided into three distinct groups: values of MPt higher than SM (group A, 4 subjects); values of MPt almost equal to SM (group B, 3 subjects); and values of MPt lower than SM (type C, 4 subjects).

\section{Discussion}

A recently developed localized MRS method, Chemical Shift Imaging (CSI), enabled obtaining typical MR spectra of the superficial (SM), and deep part of masseter (DM), and medial pterygoid muscle (MPt) from all subjects examined. So far it has been impossible to examine details of the metabolism of deep muscle, and this is the first report applying this method to the human MPt.

Prior to the study, the PCr/ $\beta$-ATP ratio of the SM, DM, and MPt of one subject were repeatedly ( 6 times) examined over 6 weeks to determine the reproducibility of the CSI results of each muscle. The relative SE [(SE/mean)x100] was $0.8 \%(\mathrm{SM}), 1.3 \%$ (DM), and $1.1 \%(\mathrm{MPt})$ (Fig. 2). The variability between these measurements is a 
combination of experimental errors and physiological variations in the subject. The result amply demonstrated the reproducibility of the measurements.

The study clearly demonstrated higher contents of PCr in the SM and MPt than in the DM both in mean values and in individual subjects. The results indicate that the SM and MPt are power producers. This is consistent with histochemical evidence that the posterior parts of the SM and MPt are characterized by a relatively high frequency (about $45 \%$ ) of large diameter type IIB fibers. Type IIB fibers belong to fast-twitching, rapidly-contracting motor units, which generate large forces and are best suited for bursts of intense, intermittent activity (Eriksson and Thornell, 1983). In contrast, DM is characterized by relatively high frequency of large type I fiber (anterior portion $72 \%$ and posterior portion $62 \%$ ). The muscle fibers of DM arranged vertically with a high concentration of complex spindles in a direction favorable to sense changes in stretch. Therefore DM is considered to be well adapted for mandibular postural control and locomotion (Eriksson and Thornell, 1983; Eriksson and Thornell, 1987).

There were no significant difference in the mean values of $\mathrm{PCr} / \beta$-ATP ratios of SM and MPt. This result is supported by MPt being a deep counterpart of masseter that acts as an elevator of the mandible with masseter, and as many similarities in these two muscles have been reported by EMG (Gibbs et al., 1984) and histochemically (Eriksson and Thornell, 1983). The study here shows a metabolic resemblance between SM and MPt.

Different from the results of the mean values, the integral values of the $\mathrm{PCr} / \mathrm{\beta}$ ATP ratios between SM and MPt varied individually. Comparing the relative amounts of $\mathrm{PCr}$ in these two muscles, the subjects could be divided into three distinct groups: values of MPt higher than SM (group A, 4 subjects); values of MPt almost equal to SM 
(group B, 3 subjects); and values of MPt lower than SM (type C, 4 subjects).

Some studies have suggested that occlusal guidance influences the EMG activity of the elevator muscles, and that group function was associated with higher elevator EMG activity (Belser and Hannam, 1985; Watanabe et al., 1998; Kahn et al., 1999), and canine guidance with lower EMG activity when the subject performs lateral movements. So we checked the occlusal conditions of all subjects as one of the factors which may influence the muscular metabolism. As a result, all group A subjects save one had less occlusal wear and canine guidance during lateral excursion of the mandible. The group B subjects all had the group function. Group C subjects all displayed pronounced occlusal wear and thus provided a distinct group function on the working side, and all of these subjects had molar balancing contact on the supporting side.

Since this study only examined the occlusal guidance (and not occlusal contact patterns or occlusal forces) of the subjects, it is not possible to evaluate details of the muscle loads with these data only. It was also not possible to determine the statistical differences between the groups. However, the results suggest that the eccentric occlusal conditions affect $\mathrm{PCr}$ amounts in the MPt, and there seems to be two possible explanations for the differences: 1) many tooth contacts in the eccentric position produce a stable platform to supply force leading to $\mathrm{PCr}$ in the MPt to produce large force at that position; or 2) PCr-rich MPt at the eccentric position causes tooth wear, and the occlusal contact increases as a result. Further detailed analysis must be made with subjects classified by occlusal condition and a statistical between group analysis is necessary to elucidate the reasons for the differences.

\section{Acknowledgment}


We wish to thank to Prof. Kazuo Miyasaka, Department of Radiology, School of

Medicine, Hokkaido University, for helpful discussion. This work was supported by a Grant-in-Aid for Scientific Research (A) (No. 09771561) from the Ministry of Education, Science and Culture, Japan.

\section{References}

Arnold DL, Taylor DJ,Radda GK (1985). Investigation of human mitochondrial myopathies by phosphorus magnetic resonance spectroscopy. Ann Neurol 18: 189-96.

Belser UC,Hannam AG (1985). The influence of altered working-side occlusal guidance on masticatory muscles and related jaw movement. J Prosthet Dent 53: 40613.

Bernus G, Gonzalez de Suso JM, Alonso J, Martin PA, Prat JA, Arus C (1993). 31PMRS of quadriceps reveals quantitative differences between sprinters and longdistance runners [see comments]. Med Sci Sports Exerc 25: 479-84.

Boicelli CA, Baldassarri AM, Borsetto C,Conconi F (1989). An approach to noninvasive fiber type determination by NMR. Int J Sports Med 10: 53-4.

Eriksson PO,Thornell LE (1983). Histochemical and morphological muscle-fibre characteristics of the human masseter, the medial pterygoid and the temporal muscles. Arch Oral Biol 28: 781-95.

Eriksson PO,Thornell LE (1987). Relation to extrafusal fibre-type composition in muscle-spindle structure and location in the human masseter muscle. Arch Oral Biol 32: 483-91.

Gibbs CH, Mahan PE, Wilkinson TM,Mauderli A (1984). EMG activity of the superior 
belly of the lateral pterygoid muscle in relation to other jaw muscles. $J$ Prosthet Dent 51: 691-702.

Kahn J, Tallents RH, Katzberg RW, Ross ME,Murphy WC (1999). Prevalence of dental occlusal variables and intraarticular temporomandibular disorders: molar relationship, lateral guidance, and nonworking side contacts. J Prosthet Dent 82: 410-5.

Meyer RA, Kuchmerick MJ,Brown TR (1982). Application of 31P-NMR spectroscopy to the study of striated muscle metabolism. Am J Physiol 242: C1-11.

Minowa K, Inoue N, Ashikaga Y, Yoshida S, Totsuka Y,Nakamura M (1998). Comparison of magnetic resonance imaging and gross findings regarding masseter muscle aponeuroses in cadavers. Oral Surg Oral Med Oral Pathol Oral Radiol Endod 86: 275-9.

Neubauer S, Horn M, Hahn D, Kochsiek K (1998). Clinical cardiac magnetic resonance spectroscopy--present state and future directions [In Process Citation]. Mol Cell Biochem 184: 439-43.

Park JH, Brown RL, Park CR, Cohn M,Chance B (1988). Energy metabolism of the untrained muscle of elite runners as observed by $31 \mathrm{P}$ magnetic resonance spectroscopy: evidence suggesting a genetic endowment for endurance exercise. Proc Natl Acad Sci U S A 85: 8780-4.

Taylor DJ, Bore PJ, Styles P, Gadian DG,Radda GK (1983). Bioenergetics of intact human muscle. A $31 \mathrm{P}$ nuclear magnetic resonance study. Mol Biol Med 1: 7794.

Vandenborne K, Walter G, Ploutz-Snyder L, Staron R, Fry A, De Meirleir K, et al. (1995). Energy-rich phosphates in slow and fast human skeletal muscle. Am J 
Physiol 268: C869-76.

Watanabe EK, Yatani H, Kuboki T, Matsuka Y, Terada S, Orsini MG, et al. (1998). The relationship between signs and symptoms of temporomandibular disorders and bilateral occlusal contact patterns during lateral excursions [In Process Citation]. J Oral Rehabil 25: 409-15. 
Table 1

Relative concentration of $\mathrm{PCr}$ in the superficial part of masseter (SM), deep part of masseter (DM), and medial pterygoid (MPt) muscles (Mean \pm SD).

\begin{tabular}{llll}
\hline & SM & DM & MPt \\
\hline PCr/ $/ \beta$-ATP & $4.68^{*} \pm 0.60$ & $3.51 \pm 0.49$ & $4.65^{*} \pm 0.75$ \\
\hline
\end{tabular}

* Significantly higher compared with deep part of masseter ( $\mathrm{p}<0.01$ by Sheffe's $\mathrm{F}$ test). 

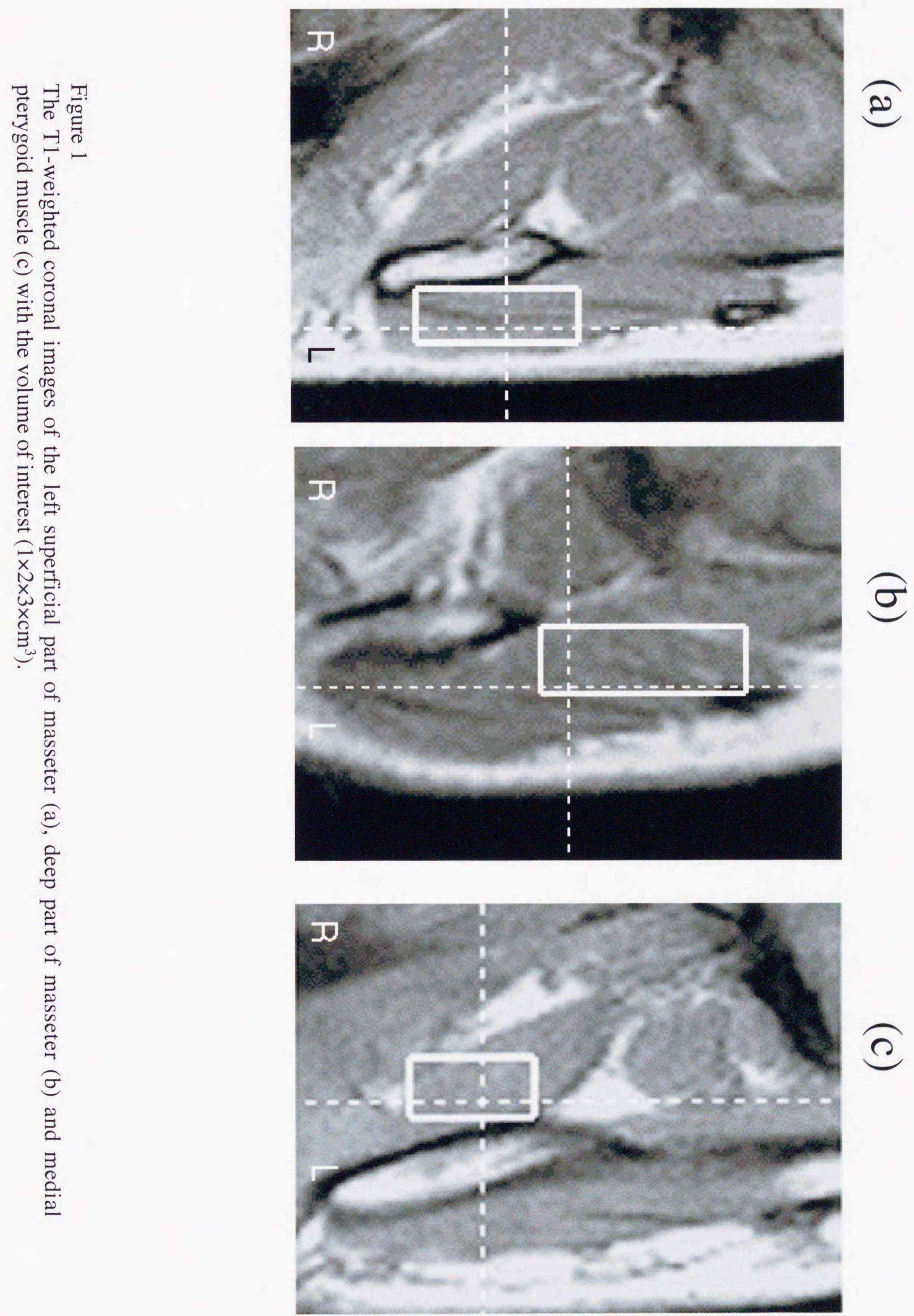


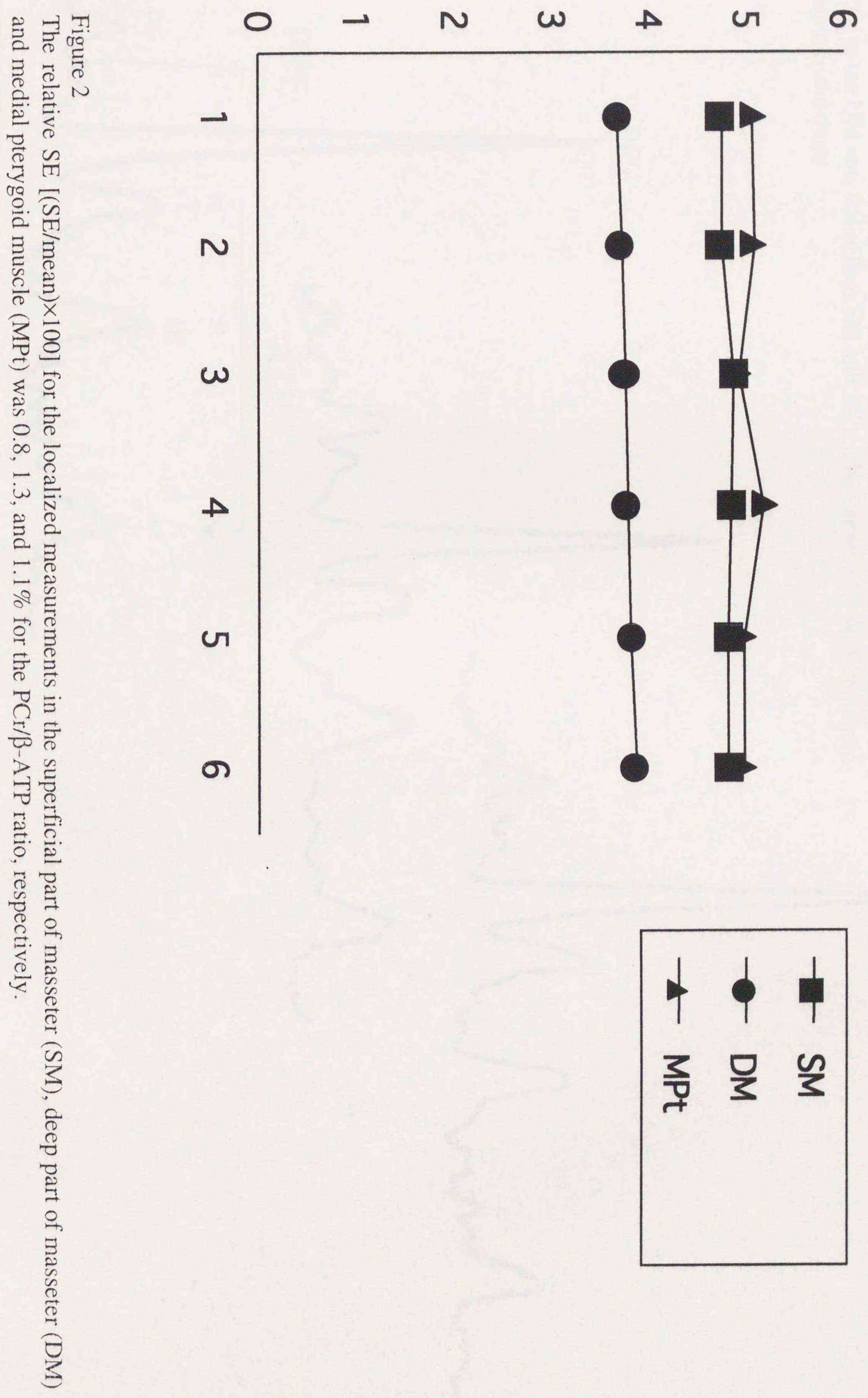




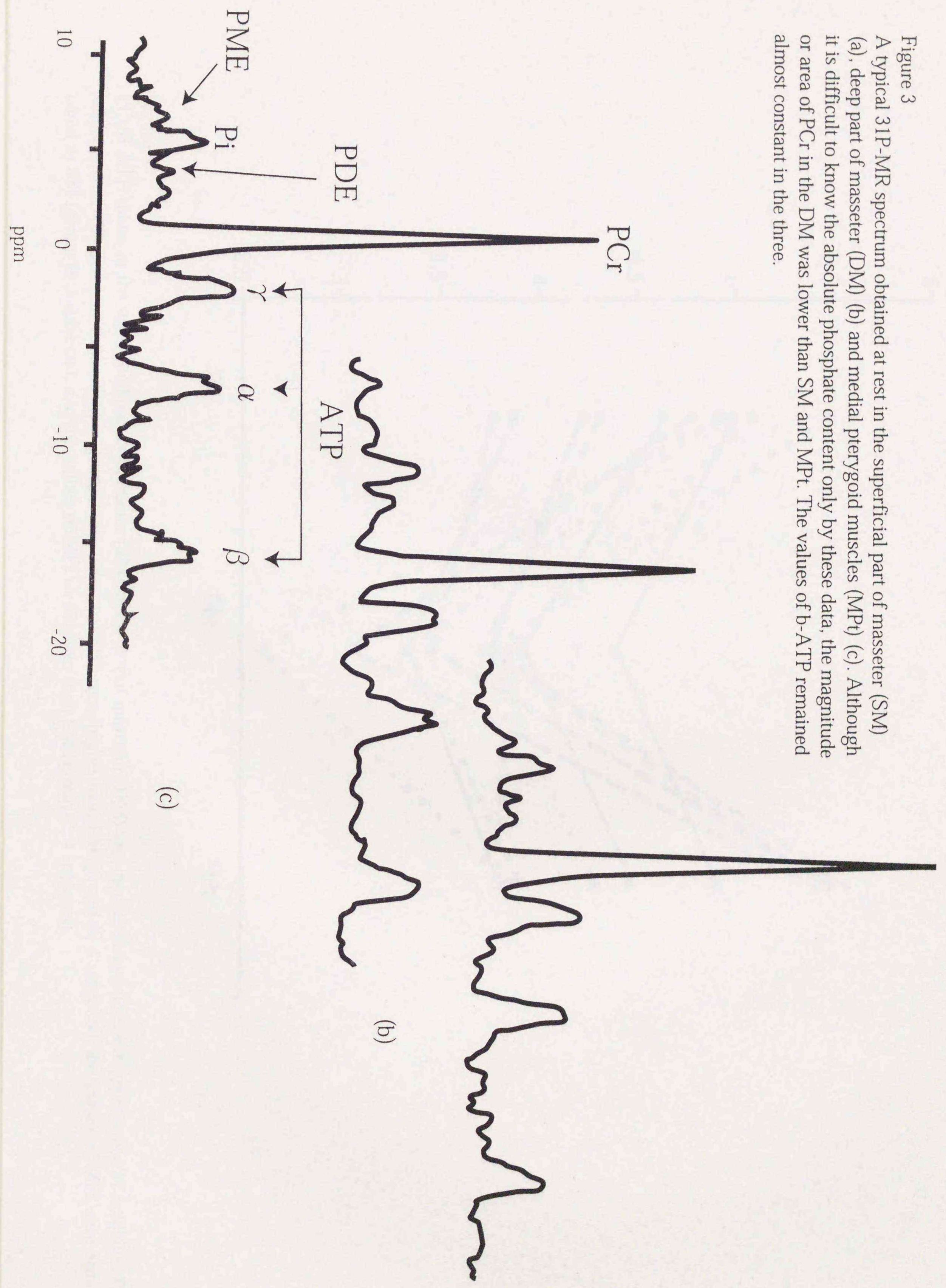




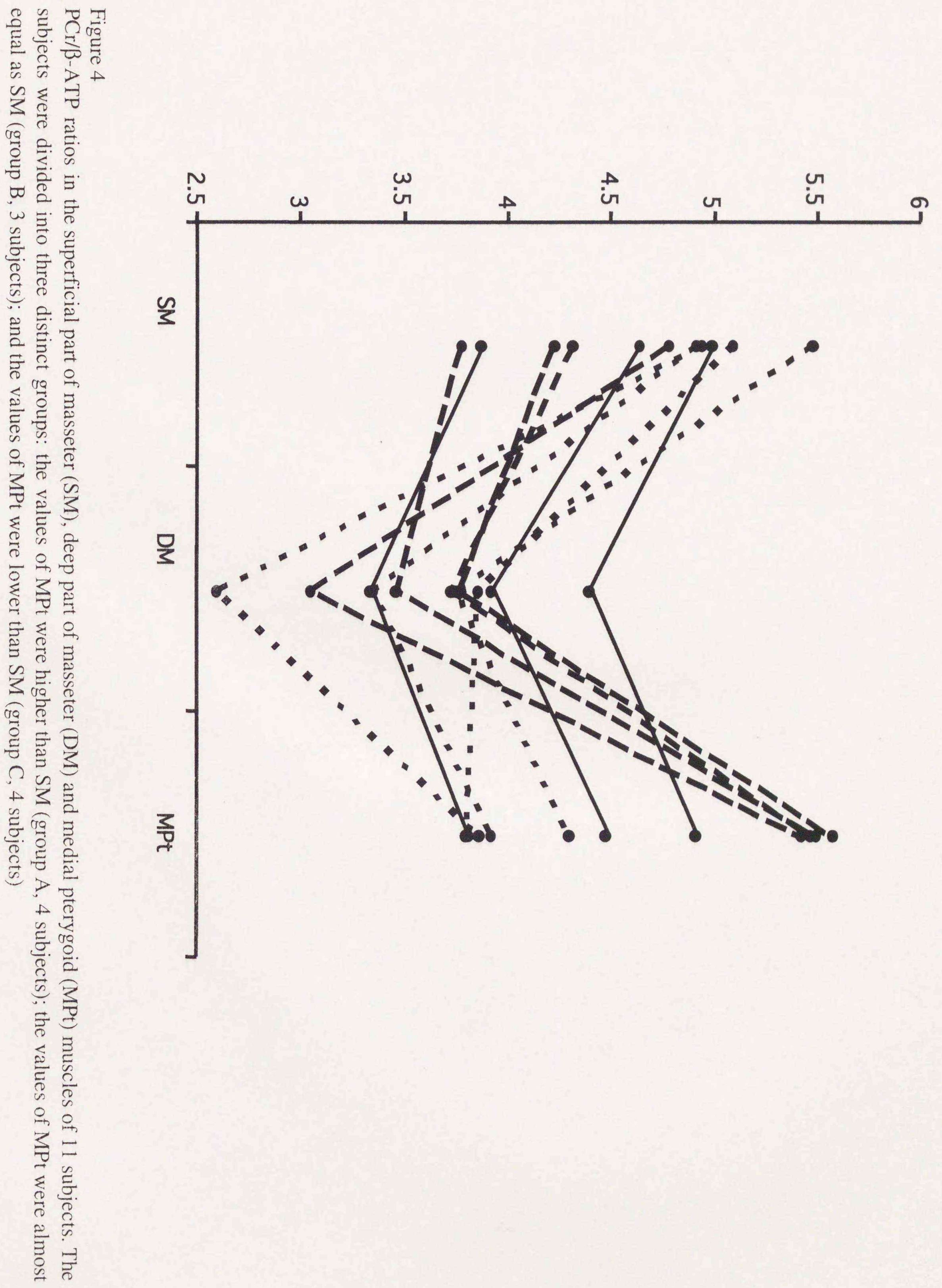





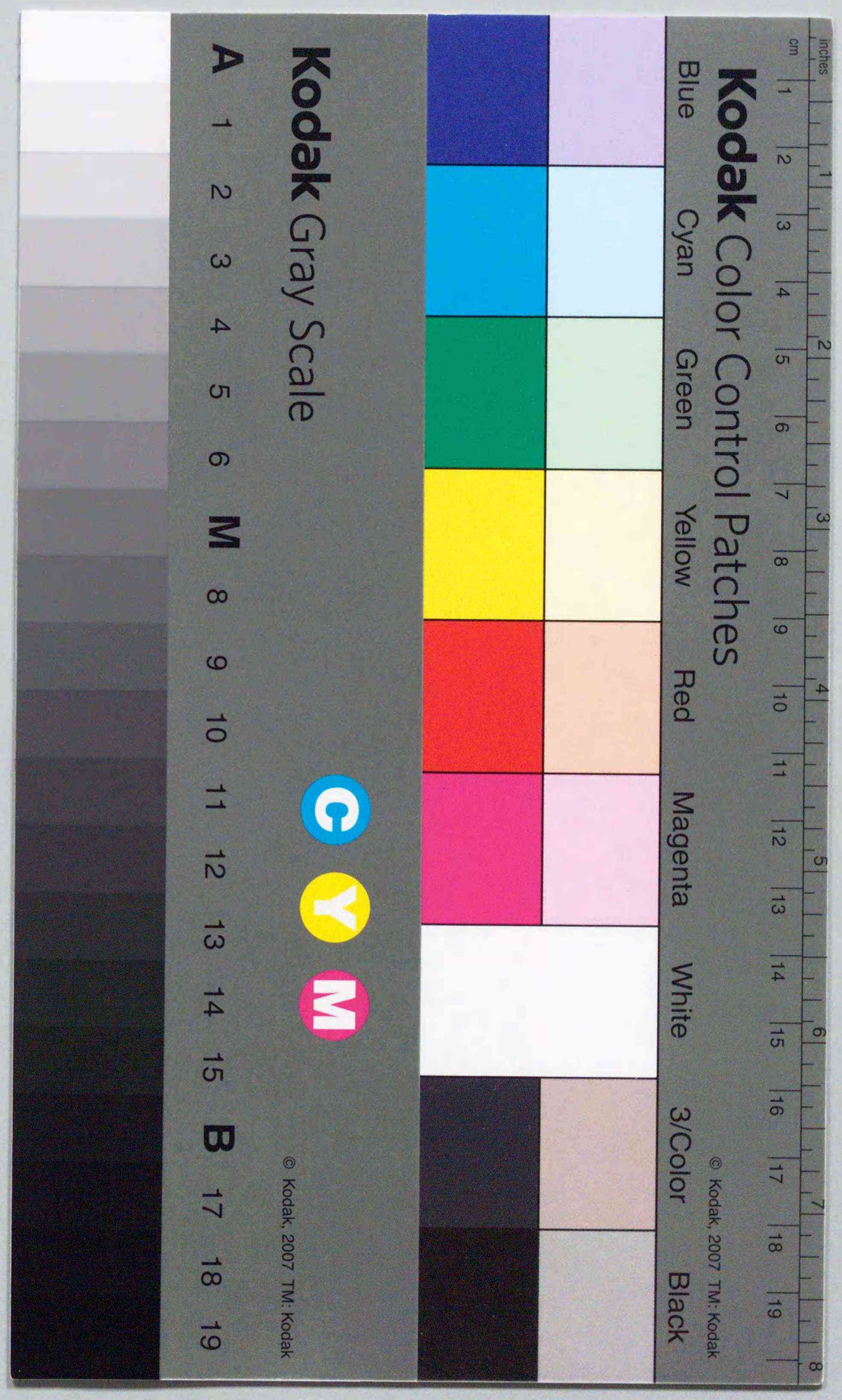

